Proceedings

\title{
Degradation Kinetic Modelling of Ascorbic Acid from Orange Juice ${ }^{+}$
}

\author{
Alina Soceanu, Nicoleta Matei *, Simona Dobrinas and Viorica Popescu
}

Citation: Soceanu, A.; Matei, N.; Dobrinas, S.; Popescu, V. Degradation Kinetic Modelling of Ascorbic Acid from Orange Juice. Proceedings 2021, 70, 55. https://doi.org/10.3390/ foods_2020-07693

Published: 9 November 2020

Publisher's Note: MDPI stays neutral with regard to jurisdictional claims in published maps and institutional affiliations.

Copyright: $@ 2020$ by the authors. Licensee MDPI, Basel, Switzerland. This article is an open access article distributed under the terms and conditions of the Creative Commons Attribution (CC BY) license (http://creativecommons.org/licenses/by/4.0/).
Department of Chemistry and Chemical Engineering, University “Ovidius” of Constanta, 124 Mamaia Blvd, 900527 Constanta, Romania; alinasoceanu@gmail.com (A.S.); sdobrinas@yahoo.com (S.D.); vpopescu@univ-ovidius.ro (V.P.)

* Correspondence: nmatei1977@yahoo.com

+ Presented at the 1st International Electronic Conference on Food Science and Functional Foods, 10-25 November 2020; Available online: https://foods_2020.sciforum.net/.

\begin{abstract}
Vitamin C or ascorbic acid is a basic nutrient, a highly effective antioxidant, widely used as food additive. Therefore, quality control in food industry demands ascorbic acid determination methods. The purpose of this study was to determine vitamin C in natural orange juices by spectrometric and voltammetric methods. Another goal was to determine the kinetic and thermodynamics activation parameters for ascorbic acid degradation in orange juices over time and at different temperatures. It was observed that during storage, ascorbic acid concentrations in orange juices were gradually decreased with time at a rate depending on storage temperature and type of orange juice. The reaction order was determined through integrated graphical analysis where the dependences of $\ln \mathrm{ct}_{\mathrm{C}} / \mathrm{C}_{0}$ as a function of time reveals the high values for $\mathrm{R}^{2}$, indicating that the kinetics of the degradation of AA follows first order reaction at both studied temperatures. For studied samples the loss of ascorbic acid was varied between $4.33 \%$ and $9.13 \%$. Enthalpy variation $(\Delta \mathrm{H})$ and entropy variation $(\Delta S)$ of activation process were obtained from the Eyring-Polany model based on transition state theory. The values of activation energy ranged between $7289.24 \mathrm{kJmol}^{-1}$ and 15689.54 $\mathrm{kJmol}^{-1}$.
\end{abstract}

Keywords: vitamin C; rate constant; activation energy; enthalpy; entropy

\section{Introduction}

A powerful antioxidant known as ascorbic acid or vitamin $C$ is a hidrosoluble vitamin present in many juices, fruits, biological systems and multivitamin preparation. It is used in treatment of cold, in cardiovascular disease, stroke and cancer [1-3].

The concentration of vitamin $C$ in beverages can be an index of quality during the storage and production process, because ascorbic acid is labile, being sensitive to the light, temperature and heat [4,5]. Many methods with different detection techniques have been reported: amperometry [3], voltammetry [5-8], spectrometry [9,10], chromatography [11], chemiluminescence [12], capillary zone electrophoresis ultraviolet detection [13]. Electrochemical techniques are the most used because of their sensitivity, selectivity, accuracy, simplicity, miniaturization and low cost. A cadmium oxide nanoparticle modified disposable screen-printed carbon electrode (SPCE) was reported by Gopalakrishnan and co. [14] for non-enzymatic detection of ascorbic acid. Also, an electrocatalytic detection of ascorbic acid using $N, N, N^{\prime}, N^{\prime}$-tetramethyl-para-phenylene-diamine (TMPD) mediated oxidation at unmodified gold electrodes was used by Sabine Kuss and Richard G. Compton [15]. The method offers sensitivity, a good limit of detection $(30 \mu \mathrm{M})$ for the detection of ascorbic acid in orange juice, without interferences from others reactants. Another voltammetric detection with modified screen-printed electrodes was studied for the determination of ascorbic acid from beverages [6]. The electrode showed a good selectivity and stability. 
For the ascorbic acid determination in fruit juices, Rodríguez-Bernaldo de Quirós et al. [2] were developed a rapid and simple HPLC method. They studied the stability of ascorbic acid during the shelf-life of the product and the degradation of about $54 \%$ was observed in a tea drink. But the cheapest way to quantify the ascorbic acid remains electrochemical methods. Amanda Silva et al. [8] were developed a square wave voltammetric method using a cobalt phthalocyanine modified carbon paste electrode for the simultaneous determination of ascorbic acid and citric, lactic, malic and tartaric acids in fruit juice without any previous pretreatment.

The purpose of this study was to determine vitamin $C$ in natural orange juices by spectrometric and voltammetric methods. Another goal was to determine the kinetic and thermodynamics activation parameters for ascorbic acid degradation in orange juices over time and at different temperatures.

\section{Results}

The analyzed samples were natural orange juices: sample 1-commercial oranges juice-with no sugar added, sample 2-commercial blood Sicilian oranges juice-with no sugar added, sample 3-commercial oranges juice with sugar and sample 4-commercial blood oranges juice with sugar.

\subsection{AA Content in Orange Juices}

In Table 1 the content of ascorbic acid in orange juices, determined by the two methods: spectrophotometric and voltammetric are presented. Determination by the voltammetric method is based on the oxidation of ascorbic acid to dehydroascorbic acid. The irreversibility of the oxido-reduction process, which takes place on the surface of the electrodes, was demonstrated using cyclic voltammetry (Figure 1).

Table 1. The content of AA mg/100 mL in studied samples.

\begin{tabular}{ccc}
\hline \multirow{2}{*}{ Sample } & \multicolumn{2}{c}{ The Content of Ascorbic Acid mg AA/100 $\mathbf{~ m L}$} \\
\cline { 2 - 3 } & Spectrometric Method & Voltammetric Method \\
\hline 1 & $29.98 \pm 0.24$ & $30.13 \pm 0.23$ \\
\hline 2 & $32.08 \pm 0.31$ & $32.13 \pm 0.37$ \\
\hline 3 & $42.14 \pm 0.43$ & $42.27 \pm 0.35$ \\
\hline 4 & $38.22 \pm 0.22$ & $38.28 \pm 0.44$ \\
\hline
\end{tabular}

Values are means, $\mathrm{n}=3$.

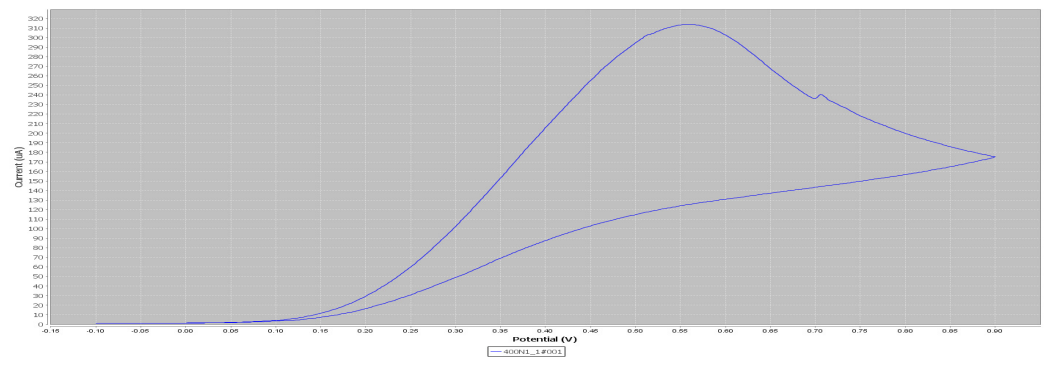

Figure 1. The cyclic voltammogram obtained for the ascorbic acid solution.

\subsection{Ascorbic Acid Degradation during Storage}

Vitamin $C$ in the orange juices was determined using the voltammetric method at different intervals of time $(0,30,60,90,120$ and $150 \mathrm{~min})$ during storage at $4^{\circ} \mathrm{C}$ and room temperature at $22{ }^{\circ} \mathrm{C}$. The degradation of ascorbic acid in orange juices was evaluated using zero-order and first-order kinetic models (Figure 2). The integrated first-order rate law over time is the Equation (1). Rate equations and the correlation coefficients $\mathrm{R}^{2}$ for the zero-order and first-order kinetic models are listed in Table 2. 
Table 2. Rate equations and the correlation coefficients.

\begin{tabular}{|c|c|c|c|c|c|}
\hline \multirow{2}{*}{ Sample } & \multirow{2}{*}{ Temperature } & \multicolumn{2}{|c|}{ Zero-Order Kinetic Model } & \multicolumn{2}{|c|}{ First-Order Kinetic Model } \\
\hline & & Rate Equation & $\mathbf{R}^{2}$ & Rate Equation & $\mathbf{R}^{2}$ \\
\hline 1 & \multirow{4}{*}{$277 \mathrm{~K}$} & $y=-0.4406 x+30.605$ & 0.9979 & $y=-0.0164 x+0.0062$ & 0.9998 \\
\hline 2 & & $y=-0.3797 x+32.607$ & 0.9919 & $y=-0.0132 x+0.007$ & 0.9998 \\
\hline 3 & & $y=-0.3734 x+42.692$ & 0.9979 & $y=-0.0094 x+0.0027$ & 0.9999 \\
\hline 4 & & $y=-0.3374 x+38.599$ & 0.9995 & $y=-0.0089 x+0.0007$ & 0.9997 \\
\hline 1 & \multirow{4}{*}{$295 \mathrm{~K}$} & $y=-0.553 x+30.657$ & 0.9992 & $y=-0.0006 x-0.0011$ & 0.9998 \\
\hline 2 & & $y=-0.52 x+32.674$ & 0.9994 & $y=-0.0006 x+0.0027$ & 0.9998 \\
\hline 3 & & $y=-0.537 x+42.815$ & 0.9998 & $y=-0.0004+0.0009$ & 0.9999 \\
\hline 4 & & $y=-0.525 x+38.829$ & 0.9989 & $y=-0.0005 x+0.0021$ & 0.9993 \\
\hline
\end{tabular}

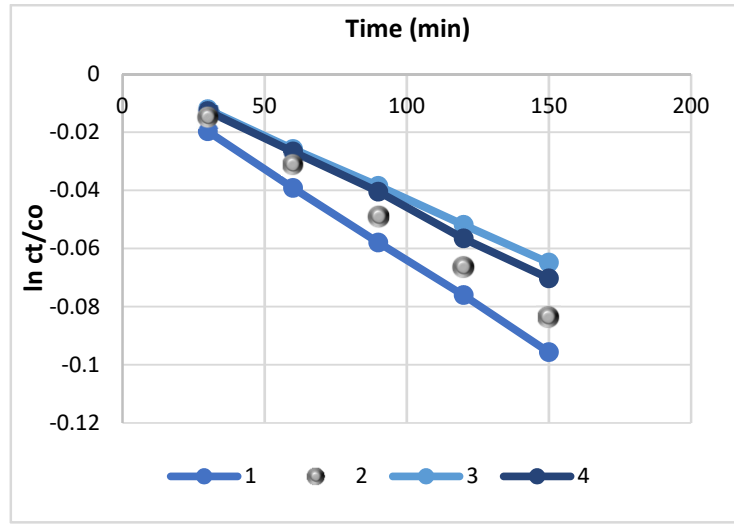

(a)

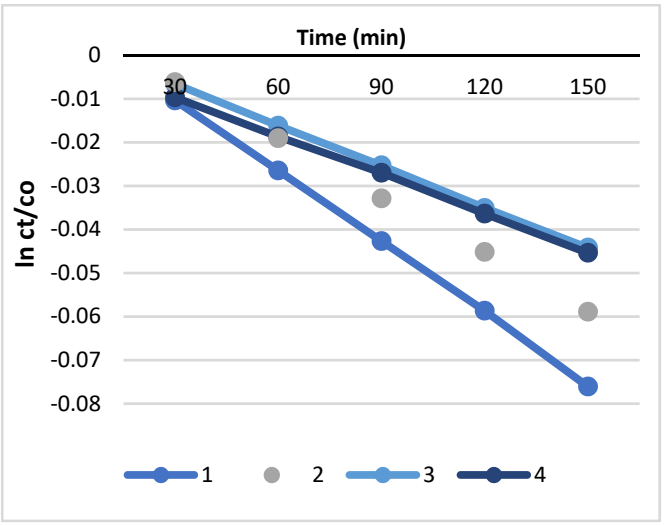

(b)

Figure 2. The dependences of $\ln \mathrm{ct} / \mathrm{co}$ as a function of time at $277 \mathrm{~K}$ (a) and $295 \mathrm{~K}$ (b).

$$
\ln \mathrm{C}-\ln \mathrm{C}_{0}=-\mathrm{k}_{1} \mathrm{t}
$$

where: $\mathrm{C}$ is the ascorbic acid concentration at time $t, \mathrm{C}_{0}$ is the ascorbic acid concentration at time $0, \mathrm{k}_{0}$ and $\mathrm{k}$ are the ascorbic acid degradation rate constant for the zero order and for the first order and $t$ is the storage time.

The first order rate constants were calculated from the slope of the straight line and the half-life time, $\mathrm{t}_{1 / 2}$ was calculated from the rate constant as $0.693 / \mathrm{k}$. The results are listed in Table 3.

Table 3. Values of rate constant and half-life time for both studied temperatures.

\begin{tabular}{|c|c|c|c|c|c|c|}
\hline Sample & Temperature & Rate Constant k (min $\left.{ }^{-1}\right)$ & $\mathbf{t}_{1 / 2}(\mathrm{~min})$ & Temperature & Rate Constant k (min $\left.{ }^{-1}\right)$ & $t_{1 / 2}(\min )$ \\
\hline 1 & \multirow{4}{*}{$277 \mathrm{~K}$} & $5 \times 10^{-4}$ & 1386 & \multirow{4}{*}{$295 \mathrm{~K}$} & $6 \times 10^{-4}$ & 1155 \\
\hline 2 & & $4 \times 10^{-4}$ & 1732.5 & & $5 \times 10^{-4}$ & 1386 \\
\hline 3 & & $3 \times 10^{-4}$ & 2310 & & $4 \times 10^{-4}$ & 1732.5 \\
\hline 4 & & $2.97 \times 10^{-4}$ & 2333 & & $4.5 \times 10^{-4}$ & 1540 \\
\hline
\end{tabular}

To calculate the activation energy value, determinations were made at different temperatures: 277 and $295 \mathrm{~K}$. To calculate activation energy, the Arrhenius equation which express the dependence of the degradation rate constant on each temperature was used. Enthalpy $\left(\Delta \mathrm{H}^{\sharp}\right)$ and entropy $\left(\Delta \mathrm{S}^{\#}\right)$ of activation were obtained from the Eyring-Polany model based on transition state theory (2):

$$
\ln \frac{k}{T}=-\frac{\Delta H \#}{R} \cdot \frac{1}{T}+\ln \frac{k_{B}}{h}+\frac{\Delta S \#}{R}
$$


where: $\mathrm{k}_{\mathrm{B}}$ is the Boltzmann's constant $\left(1.381 \times 10^{-23} \mathrm{~J} / \mathrm{K}\right) ; \mathrm{T}$ is the absolute temperature in Kelvin $(\mathrm{K})$; $\mathrm{h}$ is Planck's constant $\left(6.626 \times 10^{-34} \mathrm{Js}\right)$; $\mathrm{R}$ is the ideal gas constant $\left(8.314 \mathrm{~J} \cdot \mathrm{mol}^{-1} \mathrm{~K}^{-1}\right)$.

Activation enthalpy, $\Delta \mathrm{H}^{*}$ represents the difference in energy between the ground state and the transition state in a chemical reaction. The higher the activation enthalpy, the more energy is required for the products to form. The values for $\Delta \mathrm{H}^{\sharp}$ and $\Delta \mathrm{S}^{\sharp}$ have been determined from kinetic data obtained from $\ln \frac{k}{T}=f\left(\frac{1}{T}\right)$. The equation is a straight line with negative slope, $-\frac{\Delta H \#}{R}$ and a y-intercept, $\ln \frac{k_{B}}{h}+\frac{\Delta S \#}{R}$. The Gibbs free energy was obtain using the following thermodynamic equation:

$$
\Delta \mathrm{G}^{\sharp}=\Delta \mathrm{H}^{\sharp}-\mathrm{T} \Delta \mathrm{S}^{\sharp}
$$

The results for activation energy, activation enthalpy, activation entropy and Gibbs free energy of activation are listed in Table 4.

Table 4. Values for activation energy, activation enthalpy, activation entropy and Gibbs free energy.

\begin{tabular}{|c|c|c|c|c|c|}
\hline \multirow{2}{*}{ Sample } & \multirow{2}{*}{$\mathrm{E} \mathrm{kJmol}^{-1}$} & \multirow{2}{*}{$\Delta \mathbf{H}^{\sharp} \mathrm{Jmol}^{-1}$} & \multirow{2}{*}{$\Delta \mathbf{S}^{\ddagger} \mathbf{J} \cdot \mathbf{m o l}^{-1} \mathbf{K}^{-1}$} & \multicolumn{2}{|c|}{$\Delta \mathrm{G}^{*} \mathrm{kJmol}^{-1}$} \\
\hline & & & & $277 \mathrm{~K}$ & $295 \mathrm{~K}$ \\
\hline 1 & 7289.24 & -992.6 & -308.48 & $84,456.36$ & 90,009 \\
\hline 2 & 8422.80 & -1331.9 & -310.67 & $84,723.69$ & $90,315.75$ \\
\hline 3 & $10,856.64$ & -1868.1 & -313.60 & $84,999.1$ & $90,643.9$ \\
\hline 4 & $15,689.54$ & -2930.6 & -314.75 & $84,255.15$ & $89,920.65$ \\
\hline
\end{tabular}

\section{Discussions}

According to applied pair t-test, no statistically significant differences values of AA content determined by both methods ( $\mathrm{t}_{\text {calculated }}=0.15<\mathrm{t}_{\text {table }}=2.20$ ) were found. In the same time, $p=0.87>0.05$ which means that differences are not significant, demonstrating that the two methods: spectrometric and voltammetric can be successfully applied for the determination of ascorbic acid in the analyzed samples.

The reaction order was determined through integrated graphical analysis where the dependences of $\ln \mathrm{c}_{t} / \mathrm{c}_{0}$ as a function of time (Figure 2) reveals the high values for $\mathrm{R}^{2}$ (Table 2), indicating that the kinetics of the degradation of AA follows first order reaction at both studied temperatures. Also, the order of reaction was estimated graphically by comparing the adjusted coefficients of determination $\left(R_{a d j}^{2}\right)$ and root mean square error (RMSE) obtained from plots of AA concentration change as a function of storage time at the two temperatures for each type of juice. We selected first order reaction kinetics for which $R_{\text {adj }}^{2}$ ranged between 0.9986 to 0.9996 and RMSE ranged between 0.266 to 0.656 . On the contrary, zero-order kinetic model showed a poor performance for fitting the curve for all type of juice. It was observed that the ascorbic acid loss was decreased when the samples were kept at the refrigerator. For sample 1 the loss of ascorbic acid was: $7.33 \%$ at $277 \mathrm{~K}$ and $9.13 \%$ at $295 \mathrm{~K}$, sample $2: 5.73 \%$ at refrigerator at $8.03 \%$ at room temperature, sample $3: 4.33 \%$ at $277 \mathrm{~K}$ and $6.29 \%$ at $295 \mathrm{~K}$ and sample $4: 4.44 \%$ at refrigerator and $6.79 \%$ at room temperature. Also, the ascorbic acid loss was decreased upon sugar presence in studied juices. Oxygen is the most destructive ingredient in juice causing degradation of vitamin C. The sugar presence could decrease the concentration of dissolved oxygen in juice, thus the oxidation process of ascorbic acid was delayed. Grudić et al. [16] found that at room temperature, the ascorbic acid degradation occurs much more slowly: after 6 days the reduction of the concentration is $32 \%$ and after 12 days, the percentage is $56 \%$.

It was observed that during storage, ascorbic acid concentrations in orange juices were gradually decreased with time at a rate depending on storage temperature and type of orange juice (with or without sugar). The rate constants were increased with the increase of storage temperature. The highest rate constant was obtained for sample 1 for both studied temperatures followed by rate constant obtained for sample 2. Samples of 
blood orange juices reveal smaller values for rate constants and respectively half-life times than the orange juices, samples 1 and 2 . The rate degradation of ascorbic acid from green pepper increased with the increase of temperature and the values ranged between: $1 \times 10^{-3}$ to $5 \times 10^{-5} \mathrm{~min}^{-1}$ [16]. According to Polydera et al. [17] a notable difference in acid ascorbic degradation rates was observed when different types of packaging were used for storage of orange juice. Acid ascorbic degradation rates were almost double in case of polypropylene bottles compared to flexible pouches. The highest half-life time was obtained for sample 4 at $277 \mathrm{~K}: 2333 \mathrm{~min}$ and the smallest half-life time was obtained for sample 1 at $295 \mathrm{~K}: 1155 \mathrm{~min}$.

Activation energy ranged between $7289.24 \mathrm{kJmol}^{-1}$ and $15689.54 \mathrm{kJmol}^{-1}$. The highest values for activation enthalpy, activation entropy were obtained for sample 1 (orange juice with no sugar added). The values of thermodynamics activation parameters could be explained through the type of reaction, that is not spontaneous and it consumes energy, is endothermic. Remini et al. [18] found that the activation enthalpy and entropy for AA degradation vary with the juice type. They found that for blood orange juices values for activation enthalpy and activation entropy were ranged between $49-59 \mathrm{~kJ} / \mathrm{mol}$ and from 189 to $175 \mathrm{~J} \cdot \mathrm{mol}^{-1} \mathrm{~K}^{-1}$, respective.

\section{Materials and Methods}

Analytical grade reagents were used. L-Ascorbic acid was purchased from Merck, Darmstadt, Germany. The Fe (III) reagent was prepared by dissolving iron (III) chloride salt in distilled-deionized water and the hexacyanoferrate (III) solutions were prepared by dissolving the potassium salt in water. Metaphosphoric acid $(20 \mathrm{~g} / \mathrm{L})$ was used for extraction. Solutions of $\mathrm{H}_{2} \mathrm{SO}_{4} 0.5 \mathrm{M}$ were used for sample preparation and as supporting electrolyte because ascorbic acid oxidation is slower in acidic medium.

For spectrometric analysis, the fruit juices were sonicated, then centrifuged at 5000 rpm for 10 min, using a Hettich Universal 320 centrifuge (Andreas Hettich GmbH \& Co. $\mathrm{KG}$ ). The supernatant was filtered through filter paper (Whatman no. 42). Then, metaphosphoric acid $(20 \mathrm{~g} / \mathrm{L})$ was used for extraction. Finally, the extract was diluted with metaphosphoric acid. Blank was prepared by the same manner as except for the addition of fruit juice samples. Each of the fruit juice samples was treated separately as described under the general procedure.

Ascorbic acid in orange juices was determinate by UV-VIZ absorption spectrometry and differential pulse voltammetry (DPV) analysis with modified carbon-printed electrodes. The spectrometric method is based on the color reaction between potassium hexacyanoferrate and ascorbic acid in acid medium, when ascorbic acid has a reducing effect. The determinations were done at a wavelength of $700 \mathrm{~nm}$ using a UV-VIZ DR 3900 Lange from England spectrometer. The method was optimized in a previous paper [19]. Determination by the voltammetric method is based on the oxidation of ascorbic acid to dehydroascorbic acid. A conventional three-electrode potentiostatic system (Stat400 bipotentiostat) was used in order to carry out the electrochemical measurements. The three electrodes are integrated in the screen-printed electrodes (SPEs), where working and counter electrodes are based on a carbon ink and the pseudo-reference electrode is made up of silver. A solution of $10^{-2} \mathrm{M}$ ascorbic acid in $0.5 \mathrm{M}$ sulfuric acid was prepared and the electrochemical behavior of ascorbic acid on screen-printed electrodes was investigated.

The model's parameters were estimated by least square method. The statistical tools that were used to evaluate the adequacy of fit of the models were adjusted coefficients of determination $\left(R_{a d j}^{2}\right)$ and root mean square error (RMSE). Models with highest $R_{a d j}^{2}$ values and lowest RMSE values were used as selection criteria. To determine if the differences between values of AA content obtained by both methods (spectrometric and voltammetric) were significant, a pair t-test was performed. All statistical analyses were carried out at the $95 \%$ confidence level and two-tailed hypothesis was applied. 


\section{Conclusions}

According to applied pair t-test, were not found statistically significant differences values of AA content determined by spectrometric and voltammetric methods, demonstrating that the both methods can be successfully applied for the determination of ascorbic acid in the analyzed samples. The study revealed that the loss of ascorbic acid content increases with time and temperature. The acid ascorbic degradation in orange juice can be described by an overall first order reaction. Samples of blood orange juices reveal smaller values for rate constants and respectively half-life times than the orange juices. The highest values for activation enthalpy, activation entropy were obtained for orange juice with no sugar added.

Author Contributions: Conceptualization, A.S.; methodology, N.M, V.P.; software, S.D; validation, S.D.; formal analysis, N.M., V.P.; investigation, A.S, S.D.; resources, A.S.; data curation, A.S, S.D.; writing-original draft preparation, A.S.; writing-review and editing, S.D.; visualization, A.S, S.D.; supervision, V.P.; project administration, A.S. All authors have read and agreed to the published version of the manuscript.

Funding: This research received no external funding.

Conflicts of Interest: The authors declare no conflict of interest.

\section{References}

1. Lu, Q.; Peng, Y.; Zhu, C.; Pan, S. Effect of thermal treatment on carotenoids, flavonoids and ascorbic acid in juice of orange cv. Cara Cara. Food Chem. 2018, 265, 39-48.

2. Rodríguez-Bernaldo de Quirós, A.; Fernández-Arias, M.; López-Hernández, J. A screening method for the determination of ascorbic acid in fruit juices and soft drinks. Food Chem. 2009, 116, 509-512.

3. Uchlyama, S.; Umetsu, Y. Concentration-step amperometric sensor of L-ascorbic acid using cucumber juice. Anal. Chim. Acta 1991, 255, 53-57.

4. Aguilar, K.; Garvín, A.; Ibarz, A.; Augusto, P.E.D. Ascorbic acid stability in fruit juices during thermosonication. Ultrason. Sonochemistry 2017, 37, 375-381.

5. Guanghan, L.; Yu, W.; Leiming, Y.; Shuanglong, H. Determination of ascorbic acid in fruits and vegetables by stripping voltammetry on a glassy carbon electrode. Food Chem. 1994, 51, 237-239.

6. Florou, A.B.; Prodromidis, M.I.; Tzouwara-Karayanni, S.M.; Karayannis, M.I. Fabrication and voltammetric study of lanthanum 2,6-dichlorophenolindophenol chemically modified screen printed electrodes. Application for the determination of ascorbic acid. Anal. Chim. Acta 2000, 423, 107-114.

7. Puangjan, A.; Chaiyasith, S.; Wichitpanya, S.; Daengduang, S.; Puttota, S. Electrochemical sensor based on PANI/MnO2-Sb2 nanocomposite for selective simultaneous voltammetric determination of ascorbic acid and acetylsalicylic acid. J. Electroanal. Chem. 2016, 782, 192-201.

8. Silva, A.C.; Lourenço, A.S.; Ugulino de Araujo, M.C. Simultaneous voltammetric determination of four organic acids in fruit juices using multiway calibration. Food Chem. 2018, 266, 232-239.

9. Chan, H.S.O.; Ng, S.C.; Seow, S.H. Polybithiophene-modified electrode: Spectrophotometric detection of ascorbic acid. Synth. Met. 1994, 66, 177-183.

10. Yebra, M.C.; Cespón, R.M.; Moreno-Cid, A. Automatic determination of ascorbic acid by flame atomic absorption spectrometry. Anal. Chim. Acta 2001, 448, 157-164.

11. Zuo, R.; Zhou, S.; Zuo, Y.; Deng, Y. Determination of creatinine, uric and ascorbic acid in bovine milk and orange juice by hydrophilic interaction HPLC. Food Chem. 2015, 182, 242-245.

12. Al Mughairy, B.; Al-Lawati, H.A.J.; Eldin, F. Characterization and application of nanocolloidal Mn(IV) in a chemiluminescence system for estimating the total phenolic content in pomegranate juices using a nanodroplet microfluidics platform Suliman. Sens. Actuators B Chem. 2018, 277, 517-525.

13. Falkova, M.T.; Bulatov, A.V.; Pushina, M.O.; Ekimov, A.A.; Alekseeva, G.M.; Moskvin, L.N. Multicommutated step wise injection determination of ascorbic acid in medicinal plants and food samples by capillary zone electrophoresis ultraviolet detection. Talanta 2015, 133, 82-87.

14. Gopalakrishnan, A.; Sha, R.; Vishnu, N.; Kumar, R.; Badhulika, S. Disposable, efficient and highly selective electrochemical sensor based on Cadmium oxide nanoparticles decorated screen-printed carbon electrode for ascorbic acid determination in fruit juices. Nano Struct. Nano Objects 2018, 16, 96-103.

15. Kuss, S.; Compton, R.G. Electrocatalytic detection of ascorbic acid using $N, N, N^{\prime}, N^{\prime}$-tetramethyl-para-phenylene-diamine (TMPD) mediated oxidation at unmodified gold electrodes; reaction mechanism and analytical application. Electrochim. Acta 2017, 242, 19-24. 
16. Grudić, V.V.; Blagojević Vesna, Z.; Vukašinović-Pešić, L.; Brašanac, S.R. Kinetics of degradation of ascorbic acid by cyclic voltammetry method. Chem. Ind. Chem. Eng. Q. 2015, 21, 351-357.

17. Polydera, A.C.; Stoforos, N.G.; Taoukis, P.S. Comparative shelf life study and vitamin C loss kinetics in pasteurised and high pressure processed reconstituted orange juice. J. Food Eng. 2003, 60, 21-29.

18. Remini, H.; Mertz, C.; Belbahi, A.; Achir, N.; Dornier, M.; Madani, K. Degradation kinetic modelling of ascorbic acid and colour intensity in pasteurised blood orange juice during storage. Food Chem. 2015, 173, 665-673.

19. Matei, N.; Dobrinas, S.; Radu, G.L. Spectrophotometric determination of ascorbic acid in grapes with the Prussian Blue reaction. Ovidius Univ. Ann. Chem. 2012, 23, 174-179. 